\title{
Modelación del cambio de uso del suelo en la Zona Costera: Cuauhtemotzin-El Pailebot, Tabasco, México
}

\author{
Modeling of land use change in the Coastal Zone: Cuauhtemotzin-El Pailebot, Tabasco, Mexico
}

\section{Rodimiro Ramos Reyes}

El Colegio de la Frontera Sur, Unidad Villahermosa, Tabasco, México.

\section{Miguel Ángel Palomeque de la Cruz}

Universidad Juárez Autónoma de Tabasco, División Académica de Ciencias Biológicas, Villahermosa, Tabasco, México.

migueldacbiol@hotmail.com

\section{Héctor Javier Megia Vera}

Instituto Tecnológico Superior Villa La Venta, Huimanguillo, Tabasco.

\section{Patricia López Benítez}

El Colegio de la Frontera Sur, Unidad Villahermosa, Tabasco, México.

\section{Resumen}

En este estudio se analizaron los patrones del cambio de uso del suelo en la costa "Cuauhtemotzin-El Pailebot", Tabasco, mediante la integración de un modelo de análisis espacio temporal (Land Change Modeler) y dos modelos espaciales de predicción (Cadenas de Markov y Autómatas Celulares). Esto fue debido a la carencia de datos sobre el estado actual de la situación ambiental en la zona costera de Tabasco, a pesar de ser información relevante para sustentar escenarios futuros del cambio de uso del suelo para la planeación ambiental. Los resultados muestran durante 2000-2018 una sobresaliente pérdida de 10,644 ha del uso agropecuario, contrario a un elevado aumento de 10,210 ha de coberturas de humedales. De la misma forma, se detectó la pérdida 2,978 ha de vegetación arbórea, ante un aumento de 988 ha del área urbana. El análisis LCM de la imagen del 2018 contra la proyección 2030 detectó que las superficies agropecuarias continuaron presentaron elevadas pérdidas, ante nuevos aumentos en los humedales; mientras que la vegetación arbórea siguió presentando tasas elevadas de deforestación, con evidente crecimiento del área urbana. Los patrones adversos actuales del cambio de uso de suelo deberán ser mitigadas tomando en cuenta las proyecciones futuras mediante el planeamiento basado en el diseño de un programa de regulación ambiental y sustentable, que establezca reservas ecológicas, promueva la conservación y restauración ambiental mediante un modelo ordenamiento territorial en la zona costera de Tabasco, México.

Palabras clave: Autómatas celulares, Cadenas de Markov, Modelador del cambio de uso del suelo, Ordenamiento territorial, Zona Costera de Tabasco.

\begin{abstract}
In this study, we analyzed the patterns of land use change on the coast "Cuauhtemotzin-El Pailebot", Tabasco, by integrating a model of temporal space analysis (Land Change Modeler) and two spatial models of prediction (Markov Chains and Cellular Automata). This was due to the lack of data on the current state of the environmental situation in the coastal area of Tabasco, despite being relevant information to support future scenarios of land use change for environmental planning. The results show during 2000-2018 an outstanding loss of 10,644 ha of agricultural use, contrary to a high increase of 10,210 ha of wetland cover. In the same way, the loss 2,978 ha of tree vegetation was detected, given an increase of 988 ha of the urban area. The LCM analysis of the image of 2018 against the 2030 projection detected that agricultural areas continued to present high losses, given new increases in wetlands; while tree vegetation continued to show high rates of deforestation, with evident growth in the urban area. Current adverse patterns of land use change should be mitigated taking into account future projections through planning based on the design of an environmental and sustainable regulation program, which establishes ecological reserves, promotes environmental conservation and restoration through an orderly model territorial in the coastal area of Tabasco, Mexico.
\end{abstract}

Keywords: Cellular Automata, Markov Chains, Land Change Modeler, Environmental Planning, Tabasco Coastal Zone.

Documento recibido el 06 de noviembre de 2018 y aceptado el 25 de octubre de 2019.

Cómo citar: Ramos Reyes, R., Palomeque de la Cruz, M. A., Megia Vera, H. y López Benítez, P. (2019). Modelación del cambio de uso del suelo en la Zona Costera: Cuauhtemotzin-El Pailebot, Tabasco, México. Revista de Urbanismo, 41, 1-15. https://doi.org/10.5354/0717-5051.2019.51452 


\section{Introducción}

En el mundo los patrones más acelerados de cambios en coberturas forestales y acuáticas se dan por causas antrópicas, perdiéndose principalmente grandes coberturas de bosques tropicales (Nikokolaki, 2004; Marcantonio, Rocchini, Geri, Bacaro y Amici, 2013). Ante el deterioro ambiental que sufren las zonas tropicales, es de gran importancia conocer la distribución del cambio de uso del suelo con la finalidad de evaluar las condiciones ambientales en las que se dan los cambios e identificar las coberturas con mayor presión (Stefanov, Ramsey \& Christensen, 2001).

En México, desde la década de 1960 existe una clara tendencia de uso forestal a usos agropecuarios, donde la agricultura de temporal domina la mayoría de estas áreas (Rosete-Vergés, Pérez-Damián, Villalobos-Delgado, Navarro-Salas, Salinas-Chávez y Remond-Noa, 2014). Esto es de particular importancia, dado que la conversión de bosques tropicales a zonas agrícolas y pecuaria, representa la eliminación de sumideros de carbono, con lo cual se contribuye al cambio climático global (Mitsch et al., 2013).

Sin embargo, la conversión de tierras en el territorio mexicano, principalmente por deforestación, obedece a causas diferentes según la región, ligadas más directamente a factores ambientales, socioeconómicos y políticas públicas (Dirzo y Masera, 1996). Por ejemplo, en los años 60's un programa de colonización ordenado por el gobierno federal provocó el derribo de la selva en la península de Yucatán (Cortina Villar, Macario Mendoza y Ogneva-Himmelberger, 1999); por otro lado, la riqueza natural propia de los grandes ríos en Tabasco y parte de la Selva Lacandona en Chiapas fueron taladas con el fin de su aprovechamiento y para abrir zonas de cultivo (LópezMendoza, 1980).

No obstante, el caso de Tabasco ha sido particularmente el más drástico en términos de deforestación, por ejemplo, en tan solo 50 años se perdió casi la totalidad de la cobertura selvática con la que contaba el estado (Tudela, 1990). También se cuantificó que las selvas de Tabasco se ubican entre las más afectadas por el cambio en el uso del suelo y en el período de 1940 a 1996 se perdió el 95\% de su área, debido al incremento de los pastizales y las zonas agrícolas (Zavala y Castillo, 2007).
Las actividades económicas que se han practicado en zonas tropicales juegan un papel importante en la dinámica de uso de suelo y el deterioro ambiental. Tabasco ha presentado perdida de la vegetación y rentabilidad desde la década de 1960 por la baja capacidad productiva, que ocasionaron los cambios en las coberturas de uso de suelo y vegetación, uno de ellos es el desplazamiento de las selvas por agricultura y el boom petrolero de la década de 1970 que estimulo la economía a través de la construcción de infraestructura y el consecuente crecimiento poblacional, provocando un severo impacto en los componentes ambientales que a la fecha se pueden ver (Flores-Santiago, 1987; Gracia y Fuentes, 2004; Murillo y Martínez, 2010; Navarro y Toledo, 2008; Pinkus-Rendón y Contreras-Sánchez, 2012; Sánchez-Munguía, 2005; Tudela, 1990; Zavala et al., 2009).

Hoy en día, esos efectos son más visibles puesto que en las localidades mexicanas, el estímulo macroeconómico a través de la demanda de productos agrícolas comerciales que alcanzan elevados precios en el mercado nacional e internacional ha favorecido la rápida conversión de los usos del suelo. Tal es el caso del municipio de Huimanguillo donde la citricultura, la ganadería y los cultivos forestales (eucalipto, hule y palma de aceite) han incrementado su cobertura en las últimas décadas (Palma-López, Cisneros, Moreno, y RincónRamírez, 2007). Sin duda alguna, la modificación de las tierras de Tabasco ha sido impulsada en mayor medida por la búsqueda del crecimiento económico, tal como históricamente se ha visto en los países en vías de desarrollo (Dewan y Yamaguchi, 2009).

En las últimas décadas han aumentado las investigaciones sobre la modelación del cambio de uso del suelo en zonas tropicales, generalmente estos modelos están enfocados a estudios del ciclo, pérdida de biodiversidad o modelamiento del paisaje (Veldkamp \& Lambin, 2001). Los Sistemas de Información Geográfica son importantes para la construcción de modelos de cambio de uso del suelo, debido a la naturaleza espacial de muchas de las variables de entrada, ya que permite un manejo y análisis de los datos. En este sentido, los SIG son un aporte en: (a) la construcción de variables de entrada para la modelación; (b) la identificación de patrones espaciales; (c) la cuantificación de los cambios observados 
o predichos; (d) la evaluación de factores que operan, y; (e) la visualización de los resultados (Qi \& Wu, 1996).

Land Change Modeler está orientado al constante problema de conversión acelerada del terreno y a las necesidades analíticas bien específicas de la conservación de la biodiversidad. En LCM, las herramientas para la evaluación y la predicción de los cambios en la corteza terrestre y sus consecuencias están organizadas en torno a grandes áreas: análisis de cambios, predicción de cambios, evaluación del hábitat y su impacto sobre la biodiversidad e intervenciones de planeamiento (Khoi \& Murayama, 2011; Pontius, Huffaker \& Denman, 2004).

Las Cadenas de Markov simulan la predicción del estado de un sistema en un tiempo determinado a partir de dos estados precedentes (Eastman, 2012; Paegelow, Olmedo y Toribio, 2003). Es un procedimiento discreto en un tiempo discreto, en donde el valor en el tiempo t 1 depende de los valores en los tiempos $\mathrm{t} 0 \mathrm{y} \mathrm{t}-1$. La predicción se materializa en una serie de mapas de usos y coberturas del suelo (uno para cada categoría) para un tiempo futuro, en donde el nivel digital de cada píxel expresa la probabilidad de pertenecer a la categoría analizada (Eastman, 2012; Paegelow et al., 2003).

Los Autómatas Celulares funcionan como un conjunto de elementos idénticos, llamado células, cada una de las cuales se encuentra en un espacio discreto (Clarke \& Gaydos, 1998; Wu \& Webster, 1998). Los AC son un método de representación espacial, que se compone de reglas de transición que son aplicadas a figuras geométricas, llamadas celdas. Dichas unidades espaciales contienen una historia y una evolución de cambio en el tiempo, además de reglas como la influencia de celdas colindantes a una celda central. Este modelo se integra con la matriz de probabilidad de cambios MARKOV para crear un mapeo perfecto de la distribución espacial (Eastman, 2012).

Planteamiento del problema. En gran parte de Tabasco, la carencia de datos sobre el estado actual de la dinámica de las coberturas naturales en las zonas costeras de Tabasco aún subsiste; a pesar de ser información relevante para sustentar escenarios futuros del cambio de uso del suelo para la planeación ambiental que permitan la rehabilitación para restablecer servicios y beneficios ambientales (Palomeque-De la Cruz, Galindo-Alcántara,
Escalona-Maurice, Ruiz-Acosta, Sánchez-Martínez y PérezSánchez, 2017). Es novedosa la implementación de diversos modelos de análisis espacio temporal y modelos de predicción probabilístico que permiten describir, evaluar, analizar, explicar y predecir, en términos cuantitativos la dinámica de uso de suelo y los cambios que han ocurrido en las distintas coberturas terrestres que permiten describir el estado actual del espacio geográfico (Clarke \& Gaydos, 1998; Eastman, 2012; Paegelow et al., 2003; Wu \& Webster, 1998).

Objetivo del estudio. El objetivo de este estudio fue analizar los patrones de cambio de uso del suelo en la costa "Cuauhtemotzin - El Pailebot", Tabasco, mediante la integración de un modelo de análisis espacio temporal 2000-2018 (Land Change Modeler), y dos modelos de predicción probabilístico para proyectar el año 2030 (Cadenas de Markov y Autómatas Celulares).

Las preguntas de investigación fueron las siguientes:

¿Cuál es el cambio de uso del suelo y cuáles son las transiciones más importantes entre las coberturas naturales, y los usos antropogénicos, durante el periodo (2000-2018)?

¿Cuál es el cambio de uso del suelo y cuáles son las transiciones más importantes entre las coberturas naturales, y los usos antropogénicos, durante el 2018 contra un escenario proyectado al 2030 ?

\section{Metodología}

Área de estudio. La Zona Costera: Cuauhtemotzin-El Pailebot tiene una superficie total de 73.640 ha (Figura 1); se ubica entre las coordenadas geográficas $18^{\circ} 15^{\prime}$ y $29^{\prime \prime}$ de latitud Norte, y los $93^{\circ}$ 57' y 5.999" longitud Oeste, con temperatura media anual de $27^{\circ} \mathrm{C}$ y temperatura máxima promedia de $36^{\circ} \mathrm{C}$ (INEGI, 2006a). Gran parte de la zona pertenece a la región de la Chontalpa y tiene como cabeceras municipales a las ciudades de Huimanguillo y Cárdenas. Colinda al norte con el Golfo de México, al sur con los estados de Chiapas y Veracruz; al este con Chiapas delimitado por el río Mezcalapa; y al oeste con Veracruz, cuyo límite es el río Tonalá (Periódico Oficial, 2016). 


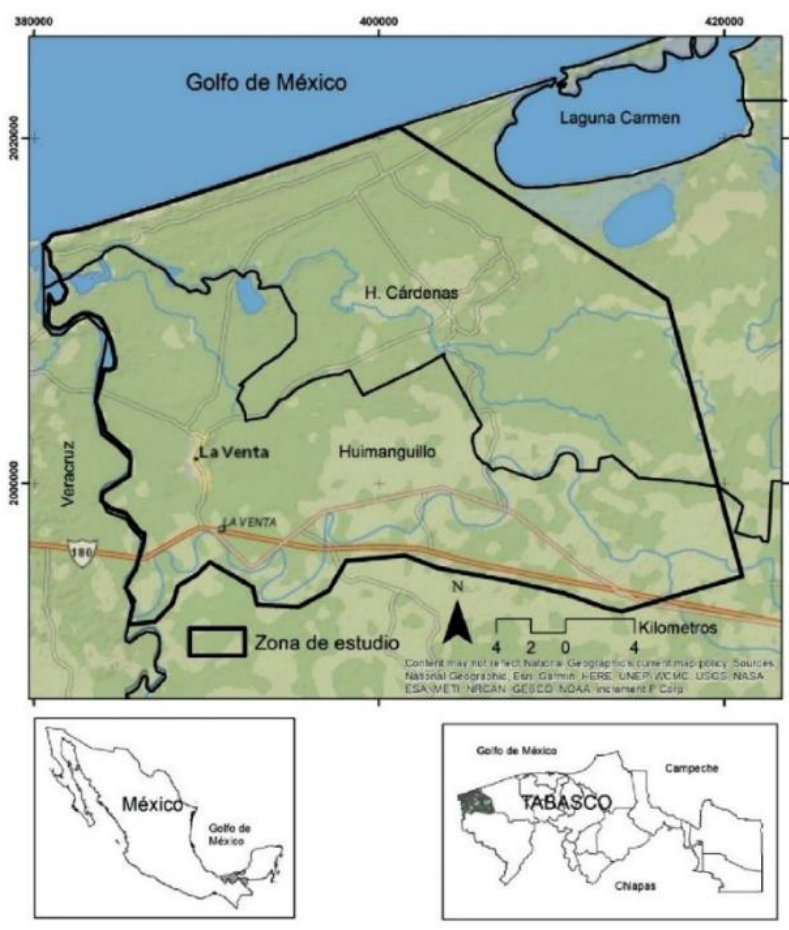

Figura 1. Área de estudio. Fuente: Elaboración propia a partir de datos de (INEGI, 2006b).

Base de datos geográfico. Se utilizaron una ortofoto del año 2000 (INEGI, 2006b) y una imagen satelital SPOT del año 2018 (INEGI, 2018). Para identificar las coberturas naturales y los principales usos de suelo, se llevó a cabo una fotointerpretación a escala de 1:15.000, aplicando los criterios de tono, forma, tamaño y textura propuestas por (Ramos-Reyes, Sánchez-Hernández y Gama-Campillo, 2016). Las categorías digitalizadas fueron: AGRAgropecuario, HUM-Humedales, ARB-Arbórea, PEMEX-
Petroleros Mexicanos, URB-Urbano. A partir de esto se crearon dos shapefile del 2000 y 2018, Datum WGS84Proyección UTM zona $15 \mathrm{~N}$, con los programas $\mathrm{QGiS}^{\circ} 3.2$ y $\operatorname{ArcGIS}^{\circ}$ 10.2. Posteriormente, los vectores fueron trasformados a formato raster con el programa IDRISI TERRSET $^{\circ}$

\section{Integración de los modelos geomáticos del cambio de uso del suelo}

Land Change Modeler 2000-2018. Para describir el cambio de uso del suelo (2000-2018) se empleó el módulo LAND CHANGE MODELER del software IDRISI TERRSET ${ }^{\circ}$, el cual genera una matriz de cambio de uso del suelo con imágenes que varían en el número de fechas de colecta; es decir, en más de dos periodos. LCM permite ubicar con gran precisión los cambios netos, las perdidas, las ganancias y las transiciones más sobresalientes (PinedaJaimes, Bosque-Sendra, Gómez-Delgado y Plata-Rocha 2009; Pontius et al., 2004; Velázquez, Mas, Gallegos, Mayorga-Saucedo, Alcántara, Castro y Palacio, 2002).

El cruce de las imágenes en LCM se validó con el módulo CROSSTAB que generó una matriz de tabulación cruzada (2000-2018) (Tabla 1) que muestran el número de pixeles que corresponden a cada combinación de categorías en las imágenes que se comparan y una matriz en términos de la proporción del número total de pixeles (2000-2018). El módulo determinó las probabilidades de V de Cramer $=0.87$ y Kappa $=0.90$. Este demostró que los mapas de 2000-2018 fueron precisos mostrando un análisis confiable de la dinámica espacial.

\section{Tabla1}

Matriz de probabilidades de cambio 2000-2018.

\begin{tabular}{llllllll}
\hline & \multicolumn{7}{c}{2018} \\
& & AGR & HUM & ARB & PEMEX & URB & Total \\
\cline { 3 - 7 } & AGR & 0,146 & 0,037 & 0,012 & 0,000 & 0,000 & 0,1957 \\
& HUM & 0,075 & 0,331 & 0,014 & 0,000 & 0,000 & 0,4240 \\
& ARB & 0,015 & 0,009 & 0,007 & 0,001 & 0,000 & 0,0310 \\
& PEMEX & 0,000 & 0,001 & 0,000 & 0,000 & 0,000 & 0,0027 \\
& URB & 0,006 & 0,001 & 0,001 & 0,000 & 0,005 & 0,0136 \\
\hline & Total & 0,2428 & 0,3799 & 0,0337 & 0,0053 & 0,0052 & 1,0000 \\
\hline
\end{tabular}

V de Cramer $=0,87$ y Kappa $=0,90$

AGR-Agropecuario, HUM-Humedales, ARB-Arbórea, PEMEX-Petroleros Mexicanos, URB-Urbano. 
Tasas de cambio. Las tasas de cambio de uso del suelo se calcularon mediante la fórmula de Palacio-Prieto et al., (2004): $T d=[(S 2 / S 1)(1 / n)-1] * 100$. Dónde: $T d=$ Tasa de cambio anual (\%), $S 1$ = Área cubierta al inicio del periodo (ha), S2 = Área cubierta al final del periodo (ha), y $n=$ Número de años del periodo.

Cadenas de Markov (2030). Se proyectó una matriz de probabilidad de transición mediante Cadenas de Markov empleando el módulo MARKOV del software IDRISI TERRSET ${ }^{\oplus}$. El método consistió en cruzar dos periodos de tiempo y simular un tercer periodo en el futuro. El módulo requirió de un error proporcional de 00 . La simulación empleó los mapas de uso del suelo 2000 y 2018, con una proyección de 12 años (2030). Los resultados de la simulación con el módulo MARKOV, fueron: (1) una matriz de transición que explica las probabilidades de cambio de uso del suelo (2030), (2) una matriz de áreas de transición en pixeles (2030), y (3) un grupo ráster para cada de las cinco categorías (2030) que enumera todas las probabilidades condicionales (Figura 2)
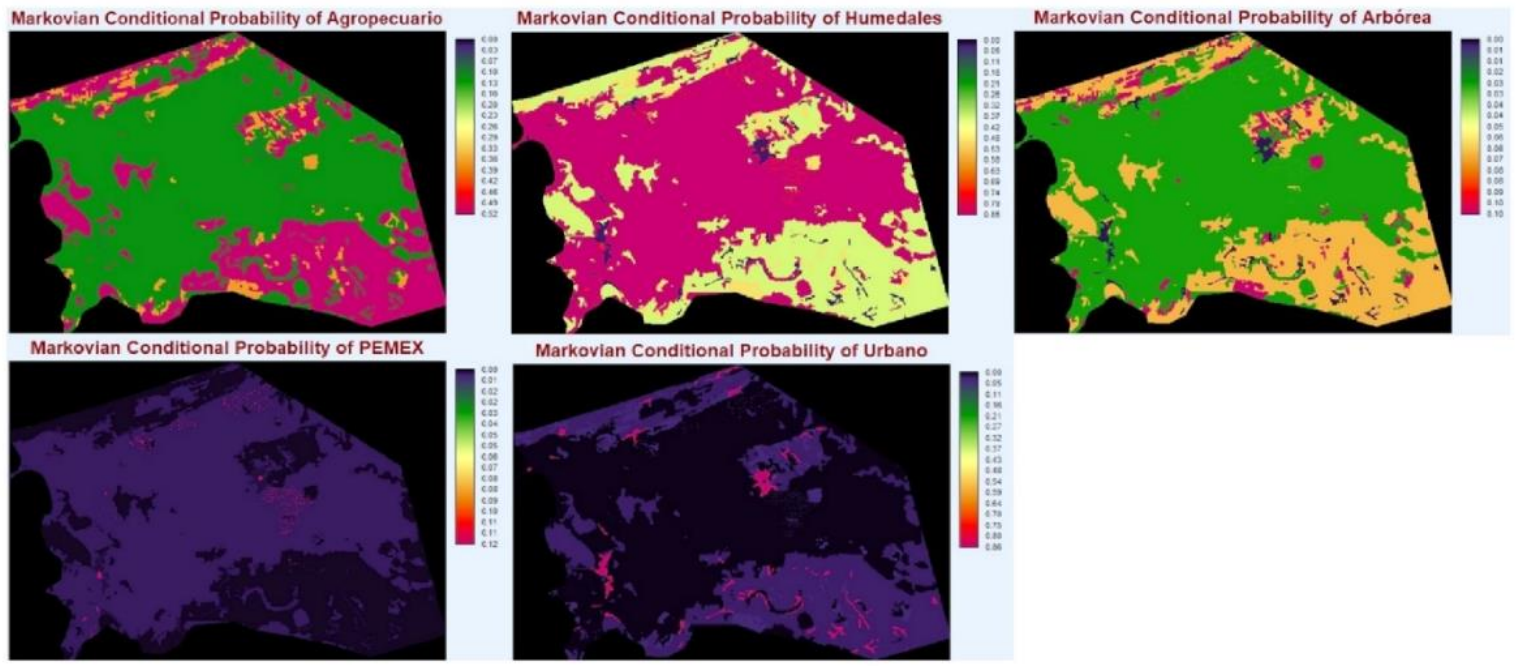

Figura 2. Imágenes de probabilidad condicionales

Autómatas Celulares (2030). Se emplearon los Autómatas Celulares, mediante el módulo CA-MARKOV del software IDRISI TERRSET ${ }^{\oplus}$ para simular un escenario espacial de uso del suelo 2030. La predicción fue construida a partir de: (1) la imagen de uso del suelo de 2018; (2) la matriz de probabilidad de transición 2030 (Tabla 5) y (3) el grupo ráster de probabilidades condicionales 2030 (Figura 2). Para validar la precisión de la imagen proyectada con CA-MARKOV, se empleó el comando VALIDATE, que calculó el estadístico Kappa (K) para indicar el grado de acuerdo entre dos mapas, ambos en sentido general y sobre una base por categoría (Eastman, 2012; Kamusoko, Aniya, Adi y Manjoro, 2009; Wang, Zheng y Zang, 2012). Para llevar a cabo este proceso, se utilizó la imagen de uso del suelo 2018 contra la proyección CA-MARKOV (2030), el resultado de esta validación fue una precisión global Kappa $=(\mathrm{Kno}=0,95 \%$, Klocation $=0,99 \%$, Kstandard=0,94\%) (Figura 3), lo cual sustenta, que la proyección 2030 es confiable para representar un escenario del cambio de uso del suelo.

\begin{tabular}{|c|c|c|c|c|c|c|}
\hline \multirow{2}{*}{\multicolumn{2}{|c|}{$\begin{array}{l}\text { Muttiples of Base Resolu } \\
\text { Information of Location }\end{array}$}} & \multirow{2}{*}{\multicolumn{2}{|c|}{$\frac{\text { Ition }(M B R): 1}{\text { No[n] }}$}} & \multicolumn{3}{|c|}{ Information of Quantity } \\
\hline & & & & Medium[m] & & Perfect[p] \\
\hline Perfect[P(x)] & & 0.5473 & & 0.9663 & & 1.0000 \\
\hline PerfectStratum[K(x)] & & 0.5507 & & 0.9663 & & 1.0000 \\
\hline MediumGrid[M[x]] & & 0.5355 & & 0.9632 & & 0.9391 \\
\hline MediumStratum[H[x]] & & 0.1667 & & 0.3354 & & 0.3301 \\
\hline $\operatorname{No}[N(x)]$ & & 0.1667 & & 0.3354 & & 0.3301 \\
\hline AgreeGridcel & $=0.6279$ & & DisagreeQuantity & $=0.0337$ & Kstandard & $=0.9447$ \\
\hline AgreeStrata & $=0.0000$ & & DisagreeStrata & $=0.0000$ & Kno & $=0.9559$ \\
\hline AgreeQuantity & $=0.1687$ & & DisagreeGridcell & $=0.0031$ & Klocation & $=0.9952$ \\
\hline AgreeChance & $=0.1667$ & & & & KlocationS & $t_{a}=0.9952$ \\
\hline
\end{tabular}

Figura 3. Validación del modelo Ca-Markov (2018/2030)

Los mapas 2018 y 2030 fueron cruzados con el módulo LCM y CROSSTAB para obtener una matriz de probabilidades de cambio validada (Tabla 2). El módulo CROSSTAB generó las probabilidades de $\mathrm{V}$ de Cramer= 
0,88 y Kappa=0,94. Este demostró que los mapas de 20182030 muestran un análisis confiable de la dinámica espacial. De este modo se calcularon los cambios netos, las perdidas, las ganancias y las transiciones más sobresalientes (Pineda et al., 2009; Pontius et al., 2004; Velásquez et al., 2002).

\section{Tabla 2}

Matriz de probabilidades de cambio 2018-2030

\begin{tabular}{llllllll}
\hline & & \multicolumn{2}{c}{2030} \\
& & AGR & HUM & ARB & PEMEX & URB & Total \\
\cline { 3 - 8 }$\infty$ & AGR & 0,1655 & 0,0000 & 0,0002 & 0,0001 & 0,0000 & 0,1658 \\
& HUM & 0,0224 & 0,4236 & 0,0035 & 0,0006 & 0,0000 & 0,4501 \\
& ARB & 0,0021 & 0,0000 & 0,0255 & 0,0000 & 0,0000 & 0,0278 \\
& PEMEX & 0,0000 & 0,0001 & 0,0000 & 0,0020 & 0,0000 & 0,0021 \\
& URB & 0,0056 & 0,0002 & 0,0017 & 0,0000 & 0,0136 & 0,0212 \\
\hline & Total & 0,1957 & 0,424 & 0,031 & 0,0027 & 0,0136 & 1,0000 \\
\hline
\end{tabular}

V de Cramer $=0,88$ y Kappa $=0,94$

AGR-Agropecuario, HUM-Humedales, ARB-Arbórea, PEMEX-Petroleros Mexicanos, URB-Urbano

\section{Resultados}

Cambio de uso del suelo (2000-2018). Con el mapa de uso del suelo del 2000 (Figura 4) se cuantificó que el uso agropecuario representaba el $36,41 \%$ del territorio. Por otra parte, las coberturas naturales como los humedales ocupaban la mayor parte de la costa con el $56,96 \%$, y la vegetación arbórea el 5,06\%. También se detectó que las áreas dedicadas a la extracción de hidrocarburos por Petróleos Mexicanos (PEMEX) ocupaban el 0,79\% y el área urbana el 0,78\% (Tabla 3).

La modelación del cambio de uso del suelo con LCM (2000-2018) (Figura 4) detectó una elevada pérdida del $9.64 \%$ del uso agropecuario con tasa negativa de $-1,2 \%$. Además, sobresale un aumento del $9.24 \%$ de coberturas de humedales. Sin embargo, el deterioro de los ecosistemas terrestres fue evidente por la elevada deforestación de la cobertura arbórea del 2,7\% que mostró una tasa negativa de $-0,5 \%$. Respecto a las actividades dedicadas a la extracción de petróleo se detectó la pérdida del $0,4 \%$, ante un aumento de $0,89 \%$ del área urbana que se posicionó como el uso antropogénico con mayor tasa de crecimiento $(5,4 \%)$ (Tabla 3).

En general, se encontró que la cobertura natural con mayor representatividad en la zona costera fue la de humedales ya que presentó una persistencia del $67,57 \%$ (Tabla 3). Contrario a esto, la vegetación arbórea no pudo mantenerse por lo que su persistencia fue de 1,38\% (Tabla 3). Cabe señalar que el uso antropogénico con mayor dominio en la costa de Tabasco es el agropecuario $(29,83 \%)$ (Tabla 3).

\section{Tabla 3}

Cuantificación del cambio de uso del suelo 2000-2018 (ha)

\begin{tabular}{cccccccccccc}
\hline Categoría & $\mathbf{2 0 0 0}$ & $\mathbf{\%}$ & $\mathbf{2 0 1 8}$ & $\mathbf{\%}$ & Ganancias & \% & Perdidas & \% & Persistencias & \% & Td(\%) \\
\hline AGR & 26.810 & 36,41 & 21,608 & 29,34 & 5.448 & 4,93 & -10.644 & $-9,64$ & 16.161 & 29,83 & $-1,2$ \\
HUM & 41.946 & 56,96 & 46.813 & 63,57 & 10.210 & 9,24 & -5.341 & $-4,84$ & 36.604 & 67,57 & 0,6 \\
ARB & 3.724 & 5,06 & 3.418 & 4,64 & 2.668 & 2,42 & -2.978 & $-2,7$ & 747 & 1,38 & $-0,5$ \\
PEMEX & 582 & 0,79 & 303 & 0,41 & 157 & 0,14 & -437 & $-0,4$ & 145 & 0,27 & $-3,6$ \\
URB & 578 & 0,78 & 1498 & 2,03 & 988 & 0,89 & -70 & $-0,06$ & 511 & 0,94 & 5,4 \\
\hline
\end{tabular}

AGR-Agropecuario, HUM-Humedales, ARB-Arbórea, PEMEX-Petroleros Mexicanos, URB-Urbano 

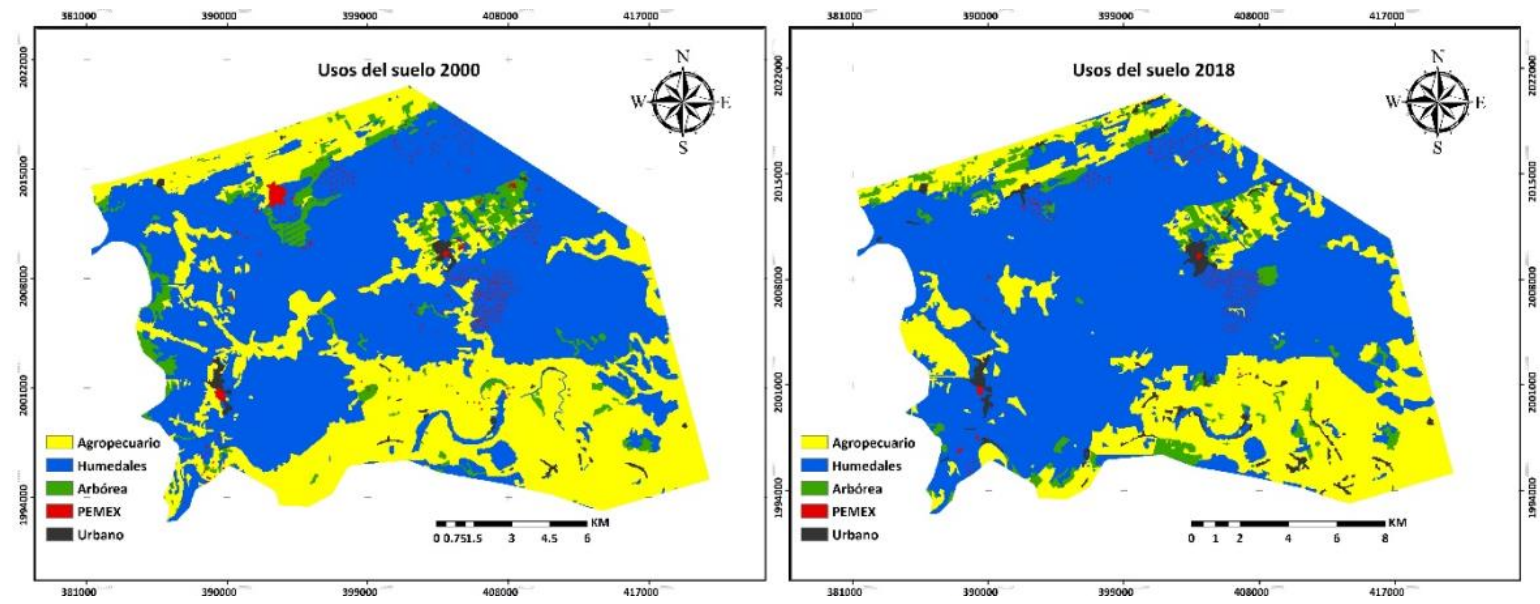

Figura 4. Mapas de uso del suelo 2000 y 2018.

Transiciones entre categorías (2000-2018). Las principales transiciones, fueron la transformación de 8.269 ha de uso agropecuario a coberturas de humedales, contrario a el cambio de 4.067 ha de humedales a uso agropecuario ( Figura 5). Otras transiciones sobresalientes fueron la conversión de 1.696 ha de superficie agropecuaria a vegetación arbórea, 1.568 ha de arbórea a humedales, 1.277 ha de arbórea a uso agropecuario, 956 ha de humedales a vegetación arbórea, y 655 ha de uso agropecuario convertidos a área urbana (Figura 5).

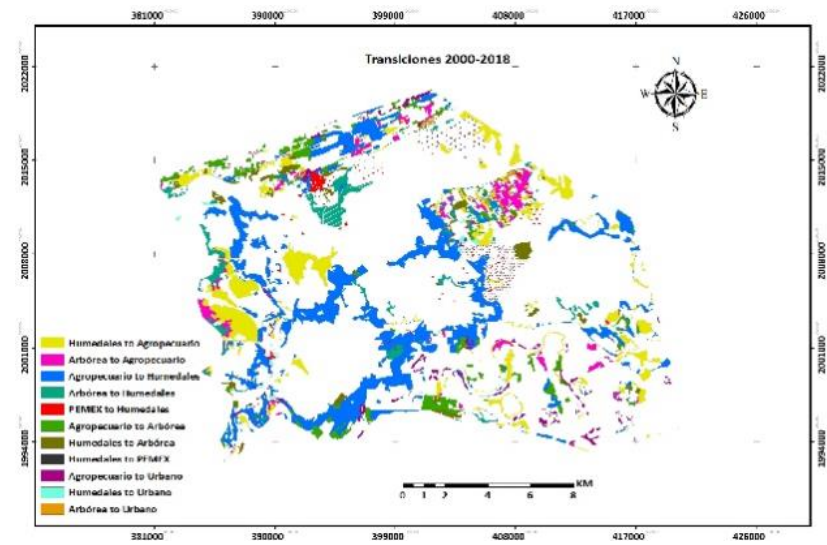

Figura 5. Mapa y grafica de las transiciones 2000-2018 (ha).

Cadenas de Markov (2030). La matriz resultante del módulo MARKOV (2030) (Tabla 4) mostró que las cinco categorías presentaron probabilidades de sufrir transiciones notables de superficie en 2030 con respecto al 2000. Esto es debido a que la modelación con Cadenas de Markov es de tipo lineal y no considera la influencia de factores externos (Pontius, 2000; Reynoso-Santos et al., 2016), sino que se basa únicamente en la dinámica interna del sistema (Paegelow et al., 2003; Reynoso-Santos,
Valdez-Lazalde, Escalona Maurice, Santos-Posadas y Pérez-Hernández, 2016).

Se obtuvo que las superficies agropecuarias presentaron probabilidad de $0,38 \%$ para transformarse en coberturas de humedales; contrario a esto, las superficies de humedales presentaron probabilidad de $0,12 \%$ para ser remplazadas por uso agropecuario (Tabla 4). De la misma forma, se detectaron remplazos de vegetación arbórea 
por uso agropecuario y por superficies de humedales con probabilidades de 0,36 y 0,49\%. También se detectó que las actividades petroleras presentaron probabilidades importantes de 0,11\% para ser remplazados en área agropecuaria. Esta matriz combinada con el modelo de Autómatas Celulares (CA-Markov) sirvió de base para la creación de la proyección espacial 2030 (Figura 6).

\section{Tabla 4}

Cadena de Markov (2030)

\begin{tabular}{lrrrrr}
\hline & AGR & HUM & ARB & PEMEX & URB \\
\hline AGR & 0.52 & 0.38 & 0.07 & 0.00 & 0.03 \\
HUM & 0.12 & 0.85 & 0.03 & 0.00 & 0.01 \\
ARB & 0.36 & 0.49 & 0.10 & 0.00 & 0.04 \\
PEMEX & 0.11 & 0.72 & 0.03 & 0.12 & 0.03 \\
URB & 0.11 & 0.03 & 0.00 & 0.00 & 0.86 \\
\hline
\end{tabular}

AGR-Agropecuario, HUM-Humedales, ARB-Arbórea, PEMEX-Petroleros Mexicanos, URB-Urbano.

Cambio de uso del suelo (2018-2030). El cruce del mapa de 2018 (Figura 4) y la proyección CA-Markov 2030 (Figura 6), detectó que las superficies agropecuarias continuaron presentaron elevadas pérdidas de 3,02\%. En relación con las coberturas naturales, los humedales continuaron ganando las superficies de $2.66 \%$ a una velocidad de cambio de $0.5 \%$, mientras que la vegetación arbórea presento nuevas pérdidas de $0.54 \%$ con una tasa de cambio negativa $-0.9 \%$ (Tabla 5 ). La presencia de actividades de extracción de petróleo (PEMEX) obtuvo perdida de $0.07 \%$, ante un continuo aumento del área urbana de $0.76 \%$ (Tabla 5 ).

\section{Tabla 5}

Cuantificación del cambio de uso del suelo 2018-2030 (ha)

\begin{tabular}{lccccccccccc}
\hline Categoría & $\mathbf{2 0 1 8}$ & $\mathbf{\%}$ & $\mathbf{2 0 3 0}$ & $\mathbf{\%}$ & Ganancias & $\mathbf{\%}$ & Perdidas & \% & Persistencias & \% & Td (\%) \\
\hline AGR & 21,608 & 36.41 & 18,306 & 24.86 & 32 & 0.03 & $-3,334$ & -3.02 & 18,273 & 26.26 & -1.4 \\
HUM & 46,813 & 56.96 & 49,698 & 67.49 & 2,932 & 2.66 & -47 & -0.04 & 46,766 & 67.21 & 0.5 \\
ARB & 3,418 & 5.06 & 3,064 & 4.16 & 245 & 0.22 & -599 & -0.54 & 2,819 & 4.05 & -0.9 \\
PEMEX & 303 & 0.79 & 235 & 0.32 & 14 & 0.01 & -81 & -0.07 & 221 & 0.32 & -2.1 \\
URB & 1,498 & 0.78 & 2,337 & 3.17 & 838 & 0.76 & 0 & 0 & 1,498 & 2.15 & 3.8 \\
\hline
\end{tabular}

AGR-Agropecuario, HUM-Humedales, ARB-Arbórea, PEMEX-Petroleros Mexicanos, URB-Urb.

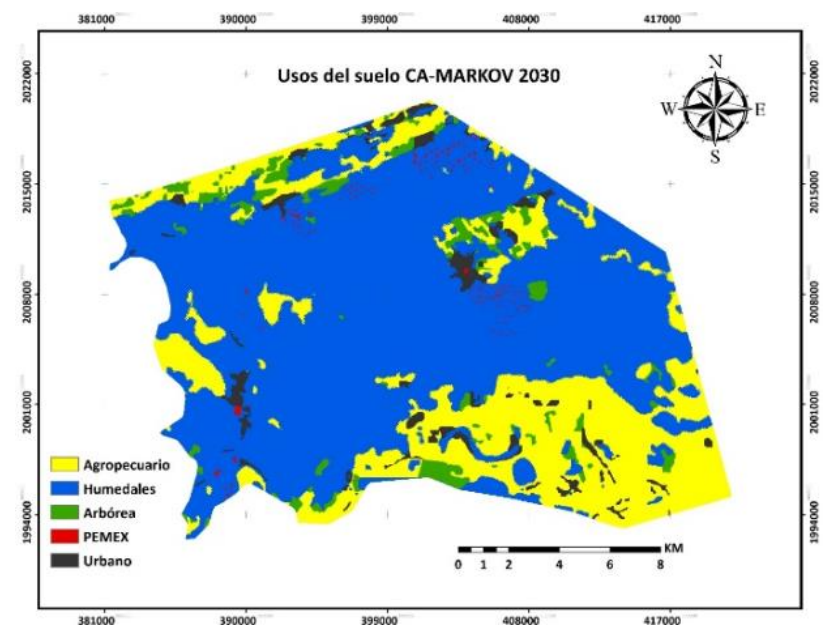

Figura 6. Mapa de uso del suelo 2030.

Transiciones entre categorías (2018 y 2030). La transición más sobresaliente de 2018-2030, fue la conversión de 2,476 ha de uso agropecuario a humedales (Figura 7). Entre otras transiciones sobresalen cambios de 389 ha de vegetación arbórea a coberturas de humedales, 237 ha de uso agropecuario a arbórea, y 185 ha de vegetación arbórea transformada a urbano (igura 7). 


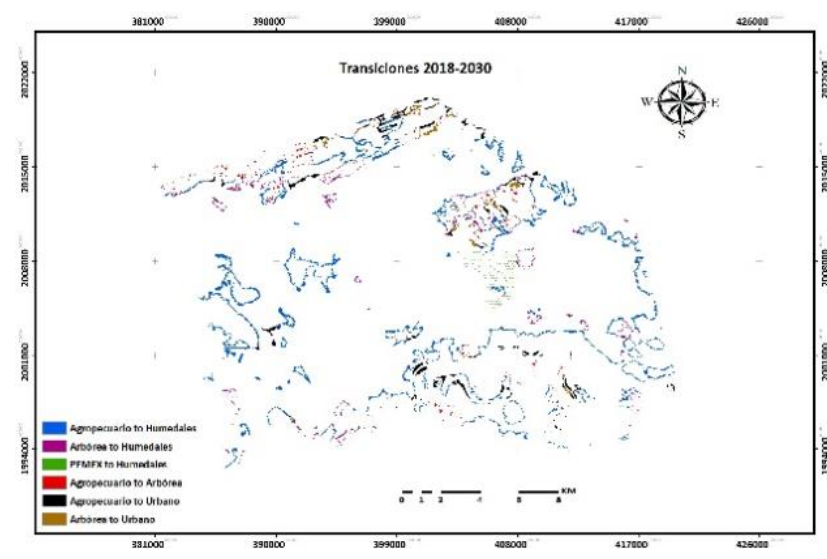

Figura 7. Mapa y grafica de las principales transiciones 2018-2030 (ha)

\section{Discusión}

El análisis espacio temporal es un referente para conocer las trayectorias pasadas, presentes y futuras de los distintos procesos de cambio en los ecosistemas que existen en determinado territorio (Mas y Flamenco, 2011). Este estudio demostró que la modelación del cambio de uso del suelo es primordial para identificar la situación ambiental de coberturas naturales en un territorio urbanizado, rural o natural, así como predecir escenarios futuros (Benítez, Pérez-Vázquez, Nava-Tablada, Equihua \& Álvarez-Palacios, 2012), y es una valiosa herramienta para proyectar impactos ambientales y socioeconómicos potenciales, y para evaluar la influencia y políticas de manejo sobre el uso del suelo (Aguayo, Pauchard, Azócar y Parra, 2009; Pijanowski, Pithadia, Shellito \& Alexandridis, 2005; Theobald \& Hobbs, 1998, Weng, 2002).

Land Change Modeler, Cadenas de Markov y Autómatas Celulares son modelos geomáticos indispensables en el estudio de los recursos naturales, y agropecuarios ya que el desarrollo, evolución y aplicación de diversas técnicas de la geomática permiten el diagnostico, evaluación y proyección, para la comprensión de los procesos clave, y para describir el futuro del uso del suelo, proporcionando oportunidades a los tomadores de decisiones, administradores y planificadores para hacer frente a futuros problemas ambientales regionales y globales (Clarke \& Gaydos, 1998; Subedi, Subedi \& Thapa, 2013). Cabe destacar que la precisión de los resultados de los modelos dependió con la correcta elaboración de los mapas de usos de suelo que expresaron una confiabilidad mayor al $90 \%$ de acuerdo al módulo CROSSTAB, por encima del porcentaje mínimo permitido para la validación cartográfica a su vez estos mapas fueron validados en campo.

El estudio comprueba que es imprescindible la integración y combinación de modelos geomáticos del cambio de uso del suelo para conocer con mayor precisión el alcance de los cambios registrados, los riesgos que ello implica y de ser posible identificar los agentes que causan el cambio con fines de seguimiento de la planificación territorial (Chuvieco, 2008). Entre otros modelos se tiene la Evaluación Multicriterio (EMC), la Regresión Múltiple y Logística, las Redes Neuronales, SLEUTH y GEOMOD (Palomeque-De la Cruz, Galindo-Alcántara, PérezSánchez, Sánchez-Martínez y Escalona-Maurice, 2017).

Para mayor precisión en el análisis de estos modelos, las variables deben coincidir en escala y los métodos de clasificación, para crear mapas de proyección de uso del suelo confiables (Velázquez et al., 2002). Cabe mencionar que estos modelos geomáticos pueden alimentarse con infinidad de variables sociales (cercanía a centros educativos, cercanía a ciudades, etc.), económicas (cercanía a centros de trabajo, industrias, carreteras, etc.), políticas (tenencia de la tierra, normatividad urbana y ambiental, etc.) y ambientales (humedales, vegetación natural, Áreas Naturales Protegidas, etc.) y físicas (relieve, pendiente y orientaciones, etc.) que proveen de mayor exactitud y realidad a la simulación (Pijanowski, Brown, Shellito \& Manik, 2002). 
Las transiciones ocurridas entre la vegetación arbórea y el uso agropecuario durante 2000-2018 están relacionados fuertemente con el proceso de conversión entre sistemas agrícolas para el periodo 2000 al 2010, principalmente en el municipio de Huimanguillo, con mayor persistencia fue el agrícola y pecuario $(83,0 \%)$ (Ramos-Reyes, Palomeque-De la Cruz, Carlos-Núñez y Sánchez-Hernández, 2019). Por otra parte, las tasas de deforestación de $-0,5 \%$ entre $2000-2018$ y 0,9\% entre 2018-2030, coinciden con estudios de Kolb y Galicia (2012) quienes observaron que la tasa de deforestación en la cuenca Grijalva-Usumacinta fue de 0,90\% durante 1993 y 2007, mientras que los informes de la FAO (Organización de las Naciones Unidas para la Alimentación y la Agricultura, 2015) reportaron tasas de deforestación en México de -0,3\% durante 1990 y 2015.

Es importante indicar que anterior al periodo de estudio (2000-2018), durante la década de 1990 la selva alta y mediana de la región de la Chontalpa, Tabasco (donde se ubica la zona costera Cuauhtemotzin-El Pailebot), ya estaba fragmentada (Flores-Santiago, 1987; Gracia y Fuentes, 2004; Murillo y Martínez, 2010; Navarro y Toledo, 2008; Pinkus-Rendón y Contreras-Sánchez, 2012; Sánchez-Munguía, 2005; Zavala et al., 2009). Las selvas de Tabasco tenían una amplia distribución en las zonas del trópico de México, aunque eran comunidades con alto índice de biodiversidad de flora y fauna, el impacto sobre este tipo de vegetación ha sido intenso (Rodríguez-Ocaña \& Banda-Izeta, 2016; Palma-López et al., 2007), debido a las características climáticas favorables para la agricultura, la cual puede llevarse a cabo sin necesidad de riego durante casi todo el año (Rodríguez-Ocaña \& Banda-Izeta, 2016).

En los años 60s los planes de modernización agropecuaria fueron los grandes causantes del deterioro ambiental de los ecosistemas de Tabasco (Navarro y Toledo, 2008; Sánchez-Munguía, 2005; Zavala y Castillo, 2007; Zavala et al., 2009), en las que destacaron; las plantaciones de plátano y cacao, orientadas al mercado internacional desde finales del siglo XIX hasta la década de 1940, la ganadería extensiva que se dirigió al mercado nacional con el Plan Chontalpa (1965-1976) y el Programa de Desarrollo Rural Integrado para el Trópico Húmedo (PRODERITH) que empezó en 1978, paralelo al auge petrolero que inició en la década de 1970 y se mantuvo hasta la década de 1980 (Capdepont-Ballina y Marín-Olán,
2014). Estos programas influyeron en la creación de nuevas zonas urbanas, aceleradas tasas de deforestación y la construcción de infraestructura hídrica, para almacenar y desviar agua en la región de las cuencas de los ríos Tonalá y Grijalva, México (Navarro y Toledo, 2008; Sánchez-Munguía, 2005; Zavala y Castillo, 2007).

Durante el auge petrolero en Tabasco (décadas de 1970 y 1980) el desarrollo económico dependió de los hidrocarburos (Allub y Michel, 1979; Lezama, 1987). En una década el sector petrolero de Tabasco aumentó 20,9\%; mientras que el sector agropecuario descendió a más de la mitad (Lezama, 1987). Sin embargo, la acelerada deforestación continuó por el incremento de zonas para actividades agropecuarias principalmente en la región Chontalpa, entre los municipios de Comalcalco y Huimanguillo (Pinkus-Rendón y Contreras-Sánchez, 2012; Zavala y Castillo, 2007). En la década de 1970, dicha deforestación redujo la selva a 146,485 ha (SánchezMunguía, 2005), de las cuales se perdieron 105,406 ha más en 1990. Esto significó que casi en cuatro décadas en Tabasco, las selvas disminuyeron del $21,7 \%$ al $1,6 \%$ (Sánchez-Munguía, 2005).

Por otra parte, las grandes ganancias en los humedales durante 2000-2018-2030 es muy favorable para el mantenimiento de los servicios ecosistémicos, por sus componentes bióticos y abióticos, como la provisión de agua y alimento, el mantenimiento de la biodiversidad de flora y fauna, regulación del clima y de las inundaciones y protección costera (Balvanera, 2012; Kandus, Morandeira y Schivo, 2010). Sin embargo, toma especial interés, el cambio de 6.216 ha de pastizal a humedales como consecuencia de los efectos del cambio climático (IPCC) representando una amenaza para la agricultura que afecta el drenaje del agua superficial y subterráneo, a cómo puede ocurrir la intrusión de agua de mar en los estuarios (Rosenzweig \& Hillel, 2008).

Ante estos procesos detectados, las tendencias de cambio de uso de suelo deben ser mitigadas mediante el planeamiento basado en el diseño de un programa de regulación urbana incluyente, transdisciplinario, transparente y sustentable, que respete el marco normativo, actualice las reservas territoriales, promueva la conservación y creación de Áreas Naturales Protegidas e incluya el ordenamiento territorial. Cabe mencionar que en el Municipio de Huimanguillo donde se ubica gran 
parte de la zona costera "Cuauhtemotzin-El Pailebot", se alertó que es primordial la elaboración de un plan municipal de ordenamiento territorial (PMOT) en donde se busque contribuir a un desarrollo sostenible; así como un Programa Municipal de Desarrollo Urbano (PMDU) donde se señalen las orientaciones de desarrollo urbano estableciendo los usos de suelo, identificando los espacios naturales y agrícolas a preservar y precisando los proyectos urbanos estratégicos (Periódico Oficial, 2016).

\section{Conclusiones}

Se analizaron los patrones del cambio de uso del suelo en la costa "Cuauhtemotzin-El Pailebot", Tabasco, mediante la integración de un modelo de análisis espacio temporal (Land Change Modeler) y dos modelos espaciales de predicción (Cadenas de Markov y Autómatas Celulares), aun con la carencia de datos sobre el estado actual de la situación ambiental en la zona costera de Tabasco, pues en la búsqueda de información se detectó que no hay antecedentes que prevean escenarios futuros del cambio de uso del suelo para la planeación ambiental.

Los resultados mostraron el evidente deterioro de los ecosistemas terrestres por la pérdida de la vegetación arbórea reflejados en sus tasas de cambio, ante un aumento del área urbana. Las principales transiciones entre las coberturas naturales y los usos del suelo entre 2000 y 2018, fueron la transformación de 8.269 ha de superficie agropecuaria a coberturas de humedales, contrario a el cambio de 4.067 ha de humedales a uso agropecuario.

El análisis espacial del 2018-2030, detectó que las superficies agropecuarias presentaron elevadas pérdidas, los humedales continuaron ganando superficies, mientras que la vegetación arbórea presento más disminuciones que ganancias, también se detectó un aumento del área urbana. La principal transición proyectada entre 2018 y 2030 de 2.476 ha de área agropecuaria a humedales.

Los resultados reafirman que el modelo de crecimiento urbano ha omitido en general la conservación de la vegetación arbórea, debido a la falta de aplicación del programa de ordenamiento del territorio. Si continua el proceso anárquico de cambio de uso del suelo por el crecimiento periurbano y crecimiento agropecuario mal planificado en la costa, la zona norte de Tabasco puede perder las últimas superficies arbóreas y acuáticas conservadas en las próximas dos décadas.

Por lo tanto, para evitar escenarios de deterioro ambiental en las próximas dos décadas, la costa "Cuauhtemotzin-El Pailebot", debe ser protegida reserva ecológica mediante el diseño de un programa de regulación urbana incluyente, transdisciplinario, transparente, sustentable y decreto legal de Áreas Naturales Protegidas, donde se promueva la conservación y restauración ambiental que incluya el ordenamiento territorial en la zona costera de Tabasco, México [B]

\section{Referencias}

Aguayo, M., Pauchard, A., Azócar, G. y Parra, O. (2009). Cambio del uso del suelo en el centro sur de Chile a fines del siglo XX: Entendiendo la dinámica espacial y temporal del paisaje. Revista chilena de historia natural, 82(3), 361-374. Recuperado de http://rchn.biologiachile.cl/pdfs/2009/3/Aguayo et al 2009.pdf

Allub, L. y Michel, M. A. (1979). La formación socioeconómica de Tabasco y el petróleo. Investigación Económica, 38(148/149), 327-355. Disponible en http://www.jstor.org/stable/42777039

Balvanera, P. (2012). Los servicios ecosistémicos que ofrecen los bosques tropicales. Revista Ecosistemas, 21(1-2). Disponible en https://www.revistaecosistemas.net/index.php/ecosi stemas/article/view/33

Benítez, G., Pérez-Vázquez, A., Nava-Tablada, M., Equihua, M. \& Álvarez-Palacios, J. L. (2012). Urban expansion and the environmental effects of informal settlements on the outskirts of Xalapa city, Veracruz, Mexico. Environment and Urbanization, 24(1), 149166. https://doi.org/10.1177/0956247812437520

Capdepont-Ballina, J. L. y Marín-Olán, P. (2014). La economía de Tabasco y su impacto en el crecimiento urbano de la ciudad de Villahermosa (1960-2010). LiminaR, 12(1), 144-160. Disponible en http:// liminar.cesmeca.mx/index.php/r1/article/view/330/3 $\underline{10}$ 
Chuvieco, E. (2000). Fundamentos de teledetección espacial. Madrid, España: Rialp.

Clarke, K. C. \& Gaydos, L. J. (1998). Loose-coupling a cellular automaton model and GIS: long-term urban growth prediction for San Francisco and Washington/Baltimore. International journal of geographical information science, 12(7), 699-714.

Cortina Villar, S., Macario Mendoza, P. y OgnevaHimmelberger, Y. (1999). Cambios en el uso del suelo y deforestación en el sur de los estados de Campeche y Quintana Roo, México. Investigaciones geográficas, (38), 41-56. Recuperado de www.investigacionesgeograficas.unam.mx/index.php /rig/article/view/59077/52064

Dewan, A. M. y Yamaguchi, Y. (2009). Land use and land cover change in Greater Dhaka, Bangladesh: Using remote sensing to promote sustainable urbanization. Applied Geography, 29(3), 390-401. https://doi.org/10.1016/j.apgeog.2008.12.005

Dirzo, R. y Masera, O. (1996). Clasificación y dinámica de la vegetación en México. En, Criterios y terminología para analizar la deforestación en México. Ciudad de México: SEMARNAP

Eastman, J. R. (2012). IDRISI Selva GIS and Image Processing Software version 17.0. Massachusetts, USA. Clark Labs.

Flores-Santiago, A. (1987). La modernización de la agricultura en el trópico húmedo mexicano: veinte años de experiencia en la Chontalpa, Tabasco. Revista de Geografía Agrícola, 13-14(2), 105-114. Disponible en

http://www.chapingo.mx/revistas/geografia/conteni do.php?id articulo=1735?id revistas $=4$ ? id revista numero $=174$

Gracia, J. y Fuentes, O. (2004). La problemática del agua en Tabasco: Inundaciones y su control. En B. Jiménez y L. Marín (Eds.), El agua en México vista desde la academia (pp. 177-185). Ciudad de México: Academia Mexicana de Ciencias.

Instituto Nacional de Estadística, Geografía e Informática (2006a). Síntesis geográfica y anexo Cartográfico del Estado de Tabasco. Aguascalientes: INEGI.

Instituto Nacional de Estadística, Geografía e Informática (2006b). Fotografía aérea - INEGI. Aguascalientes: INEGI.

https://www.inegi.org.mx/temas/imagenes/fotoaere a/areahistorica/
Instituto Nacional de Estadística, Geografía e Informática (2018). Estación de Recepción México de la Constelación SPOT. Aguascalientes: INEGI Instituto Nacional de Estadística, Geografía e Informática. Recuperado de https://www.inegi.org.mx/400.html?aspxerrorpath=/ geo/contenidos/imgpercepcion/imgsatelite/estacion recepcion.aspx

Kamusoko, C., Aniya, M., Adi, B. \& Manjoro, M. (2009). Rural sustainability under threat in Zimbabwesimulation of future land use/cover changes in the Bindura district based on the Markov-cellular automata model. Applied Geography, 29(3), 435-447. https://doi.org/10.1016/j.apgeog.2008.10.002

Kandus, P. Morandeira, N. y Schivo, F. (2010). Bienes y servicios ecosistémicos de los humedales del Delta del Paraná. Buenos Aires: Wetlands InternationalFundación Humedales.

Khoi, D. D. \& Murayama, Y. (2011). Modeling deforestation using a neural network-Markov model. In Spatial Analysis and Modeling in Geographical Transformation Process (pp. 169-190). Dordrecht: Springer.

Kolb, M. y Galicia, L. (2012). Challenging the linear forestation narrative in the Neo-tropic: Regional patterns and processes of deforestation and regeneration in southern Mexico. The Geographical Journal, 178(2), 147- 161. https://doi.org/10.1111/j.1475-4959.2011.00431.x

Lezama, J. L. (1987). Migración y petróleo en Tabasco. Estudios Demográficos y Urbanos, 2(2), 231-256. Disponible en http://www.jstor.org/stable/40314407

López-Mendoza, R. (1980). Tipos de vegetación y su distribución en el estado de Tabasco y norte de Chiapas. Texcoco, México: Universidad Autónoma Chapingo

Marcantonio, M., Rocchini, D., Geri, F., Bacaro, G. y Amici, V. (2013). Biodiversity, roads, \& landscape fragmentation: Two Mediterranean cases. Applied Geography, 42, 63-72. https://doi.org/10.1016/j.apgeog.2013.05.001

Mas, J. F. y Flamenco, A. (2011). Modelación de los cambios de coberturas/uso del suelo en una región tropical de México. GeoTrópico, 5(1), 1-24. Disponible en http://www.geotropico.org/NS 5 1 MasFlamenco.pdf 
Mitsch, W. J., Bernal, B., Nahlik, A. M., Mander, Ü., Zhang, L., Anderson, C. J. y Brix, H. (2013). Wetlands, carbon, and climate change. Landscape Ecology, 28(4), 583-597.

https://link.springer.com/article/10.1007/s10980012-9758-8

Murillo, L. D. y Martínez, R. J. (2010). Comunicación para el desarrollo en México: reflexiones sobre una experiencia en el trópico húmedo. Estudios sobre las culturas contemporáneas, (31), 201- 225. Recuperado de

http://www.culturascontemporaneas.com/contenido s/8\%20Testimonio\%20 Murillo\%20pp\%20201-225.pdf

Navarro, J. M. y Toledo, H. (2008). Transformación de la cuenca del río Grijalva, Revista Noticias AMIP, 4(16), 11-22. Disponible en http://www.amip.org.mx/htm/RevAMIP/A4NUM16/

Nikolakaki, P. (2004). A GIS site-selection process for habitat creation: estimating connectivity of habitat patches. Landscape and urban planning, 68(1), 77-94. https://doi.org/10.1016/S0169-2046(03)00167-1

Organización de las Naciones Unidas para la Alimentación y la Agricultura (FAO). (2015). Evaluación de los recursos forestales mundiales 2015. Roma, Italia: Autor. Disponible en http://www.fao.org/3/ai4808s.pdf

Paegelow, M., Olmedo, M. C. y Toribio, J. M. (2003). Cadenas de Markov, evaluación multicriterio y evaluación multiobjetivo para la modelización prospectiva del paisaje. GeoFocus, (3), 22-44. Recuperado de http://www.geofocus.org/index.php/geofocus/article /view/21

Palacio-Prieto, J. L., Sánchez-Salazar, M. T., Casado, J. M., Propin, F. E., Delgado, C. J. y Velázquez, M. A. (2004). Indicadores para la caracterización y el ordenamiento territorial. México, D.F: SEMARNAT

Palma-López, D. J., Cisneros, D. J., Moreno, C. E. y RincónRamírez, J. A. (2007). Suelos de Tabasco: su uso y manejo sustentable. Villahermosa, México: Colegio de Postgraduados

Palomeque-De la Cruz, M. Á., Galindo-Alcántara, A., Escalona-Maurice, M. J., Ruiz-Acosta, S. D. C., Sánchez-Martínez, A. J. y Pérez-Sánchez, E. (2017). Analysis of land use change in an urban ecosystem in the drainage area of the Grijalva river, Mexico. Revista Chapingo. Serie Ciencias Forestales y del Ambiente, 23(1). http://dx.doi.org/10.5154/r.rchscfa.2016.03.018.
Palomeque-De la Cruz, M. Á., Galindo-Alcántara, A., Pérez-Sánchez, E., Sánchez-Martínez, A. J. y EscalonaMaurice J. M. (2017). Modelos geomáticos con base en transición para el análisis espacial en Villahermosa, Tabasco. Revista mexicana de ciencias agrícolas, 8(2), 253-267. Disponible en http://www.scielo.org.mx/scielo.php?pid=S2007$\underline{09342017000200253 \& \text { script=sci arttext }}$

Periódico Oficial. (2016). Plan Municipal de Desarrollo 2016-2018. Huimanguillo, Tabasco. México: Gobierno del estado de Tabasco.

Pijanowski, B. C., Brown, D. G., Shellito, B. A. y Manik, G. A. (2002). Using neural networks and GIS to forecast land use changes: a land transformation model. Computers, environment and urban systems, 26(6), 553-575. https://doi.org/10.1016/S01989715(01)00015-1

Pijanowski, B. C., Pithadia, S., Shellito, B. A. y Alexandridis, K. (2005). Calibrating a neural networkbased urban change model for two metropolitan areas of the Upper Midwest of the United States. International Journal of Geographical Information Science, 19(2), 197-215. https://doi.org/10.1080/13658810410001713416

Pineda-Jaimes, N. B., Bosque-Sendra, J., Gómez-Delgado, M. y Plata-Rocha, W. (2009). Análisis de cambio del uso del suelo en el Estado de México mediante sistemas de información geográfica y técnicas de regresión multivariantes: Una aproximación a los procesos de deforestación. Investigaciones geográficas, (69), 33-52 Recuperado de http://www.scielo.org.mx/scielo.php?script=sci_artte xt\&pid=S0188-46112009000200004

Pinkus-Rendón, M. J. y Contreras-Sánchez, A. (2012). Impacto socioambiental de la industria petrolera en Tabasco: el caso de la Chontalpa. LiminaR, 10(2), 122144. Disponible en http://liminar.cesmeca.mx/index.php/r1/article/view /99/86

Pontius, R. G. (2000). Quantification error versus location error in comparison of categorical maps. Photogrammetric Engineering and Remote Sensing. 66(8):1011-1016. Disponible en https://www2.clarku.edu/faculty/rpontius/pontius 2 000 pers.pdf

Pontius Jr, R. G. Huffaker, D. y Denman, K. (2004). Useful techniques of validation for spatially explicit landchange models. Ecological Modelling, 179(4), 445461. https://doi.org/10.1016/j.ecolmodel.2004.05.010 
Qi, Y. y Wu, J. (1996). Effects of changing spatial resolution on the results of landscape pattern analysis using spatial autocorrelation indices. Landscape ecology, 11(1), 39-49. Disponible en https://link.springer.com/article/10.1007/BF0208711 $\underline{2}$

Ramos-Reyes, R., Palomeque-De la Cruz, M., CarlosNúñez, J. y Sánchez-Hernández, R. (2019). Análisis geomático espacial del cambio de uso del suelo en Huimanguillo, Tabasco (2000-2010-2030). Revista Mexicana De Ciencias Forestales, 10(53). https://doi.org/https://doi.org/10.29298/rmcf.v10i53 .555

Ramos-Reyes, R., Sánchez-Hernández, R. y GamaCampillo, L. M. (2016). Análisis de cambios de uso del suelo en el municipio costero de Comalcalco, Tabasco, México. Ecosistemas y recursos agropecuarios, 3(8), 151-160. Disponible en http://www.scielo.org.mx/scielo.php?pid=S2007$\underline{90282016000200151 \& \text { script=sci arttext\&tlng=en }}$

Reynoso-Santos, R. Valdez-Lazalde, J. R. Escalona Maurice, M., Santos-Posadas, H. M. y PérezHernández, M. J. 2016. Cadenas de Markov y autómatas celulares para la modelación de cambio de uso de suelo. Ingeniería hidráulica y ambiental, 37(1), 72-81. Recuperado de http://scielo.sld.cu/pdf/riha/v37n1/riha06116.pdf

Rodríguez-Ocaña, L. \& Herminia-Banda, I. (2016). Ecotourism in water jungle Tabasco, Mexico: media promotion. International Journal of Scientific Management and Tourism, 2(3), 291-306. Recuperado de http://ijosmt.com/index.php/ijosmt/article/view/141

Rosenzweig, C. \& Hillel, D. (2008). Climate variability and the global harvest: Impacts of El Niño and other oscillations on agro-ecosystems. New York: Oxford University Press.

Rosete-Vergés, F. A., Pérez-Damián, J. L., VillalobosDelgado, M., Navarro-Salas, E. N., Salinas-Chávez, E. y Remond-Noa, R. (2014). El avance de la deforestación en México 1976-2007. Madera y bosques, 20(1), 2135. Recuperado de http://www.scielo.org.mx/pdf/mb/v20n1/v20n1a3.p $\underline{\mathrm{df}}$

Sánchez-Munguía, A. (2005). Uso del suelo agropecuario y deforestación en Tabasco 1950-2000. Villahermosa: Universidad Juárez Autónoma de Tabasco.
Stefanov, W. L., Ramsey, M. S. \& Christensen, P. R. (2001). Monitoring urban land cover change: An expert system approach to land cover classification of semiarid to arid urban centers. Remote sensing of Environment, 77(2), 173-185. https://doi.org/10.1016/S0034-4257(01)00204-8

Subedi, P., Subedi, K. \& Thapa, B. (2013). Application of a hybrid cellular automaton-Markov (CA-Markov) model in land-use change prediction: a case study of Saddle Creek Drainage Basin, Florida. Applied Ecology and Environmental Sciences, 1(6), 126-132.

Theobald, D. M. \& Hobbs, N. T. (1998). Forecasting rural land-use change: a comparison of regression-and spatial transition-based models. Geographical and Environmental Modelling, 2, 65-82. Disponible en https://www.researchgate.net/publication/27990097 7 Forecasting rural landuse change a comparison of regressionand spatial transition-based models

Tudela, F. (1990). La modernización forzada del trópico: el caso de Tabasco. Ciudad de México: El Colegio de México.

Velázquez, A., Mas, J. F., Gallegos, J. R. D., MayorgaSaucedo, R., Alcántara, P. C., Castro, R. y Palacio, J. L. (2002). Patrones y tasas de cambio de uso del suelo en México. Gaceta ecológica, (62), 21-37. Recuperado de

https://scholar.google.com.mx/scholar?hl=es\&as sdt $=0 \% 2 \mathrm{C} 5 \& \mathrm{q}=$ Patrones+y+tasas+de+cambio+de+uso+d el+suelo+en+M\%C3\%A9xico\&btnG=

Veldkamp, A. \& Lambin, E.F. (2001). Predicting land-use change. Agriculture, Ecosystems, and Environment, 85 (1-3): 1-6. https://doi.org/10.1016/S01678809(01)00199-2

Wang, S. Q., Zheng, X. Q. \& Zang, X. B. (2012). Accuracy assessments of land use change simulation based on Markov-cellular automata model. Procedia Environmental Sciences, 13, 1238-1245. https://doi.org/10.1016/j.proenv.2012.01.117

Weng, Q. (2002). Land use change analysis in the Zhujiang Delta of China using satellite remote sensing, GIS and stochastic modelling. Journal of environmental management, 64(3), 273-284. https://doi.org/10.1006/jema.2001.0509

Wu, F. \& Webster, C. J. (1998). Simulation of land development through the integration of cellular automata and multicriteria evaluation. Environment and Planning B: Planning and design, 25(1), 103-126. https://doi.org/10.1068/b250103 
Zavala, J., Castillo, A. O., Ortiz, I. C., Palma, D. J., Salgado, G. S., Rincón, R. J. A. y Ramos, R. R. (2009). Capacidad de uso del suelo urbano en Tabasco: Con base en suelo, uso actual y vegetación. Cárdenas: Colegio de Posgraduados.
Zavala, J. y Castillo, A. O. (2007). Cambio de uso de la tierra en el estado de Tabasco. En D. J. Palma y A. Triano (Eds.), Plan de uso sustentable de los suelos del estado de Tabasco (vol, II, pp. 38-56). Villahermosa: Colegio de Posgraduados. 